\title{
A CASE OF FEVER OF UNKNOWN ORIGIN AND ABDOMINAL MASS
}

\author{
AHMED $\mathrm{M}^{1}$, PATWARY MI ${ }^{2}$, ANSARI MAJ ${ }^{3}$, CHOUDHURY MSR 4
}

\begin{abstract}
:
A 42 year old man was admitted to hospital with the complaints of low grade fever, gradual weight loss and pigmentation of his skin for about one year. On physical examination, the patient who was to be a thin, slightly emaciated man, had low grade fever along with pigmentation all over his body. Serum electrolytes done repeatedly showed mild hyponatraemia with sodium $<135 \mathrm{mmol} / \mathrm{L} \&$ persistent hyperkalaemia $\left(k^{+}: 5-5.5 \mathrm{meq} / \mathrm{L}\right)$. Ultrasonogram and computed tomography (CT) scan of abdomen revealed bilateral adrenal mass. Subsequently the FNAC of adrenal gland done in a recognized hospital in Dhaka was suggestive of adrenal tuberculosis. The patient was started on four-drug anti-tuberculosis therapy (isoniazid, rifampicin, pyrazinamide and ethambutol) along with hydrocortisone supplementation. On completion of treatment the patient became asymptomatic with marked improvement in skin pigmentation.
\end{abstract}

Key words: Addison's disease, fever of unknown origin, bilateral adrenal mass, adrenal tuberculosis, tuberculous Addison's disease

J Dhaka Med Coll. 2011; 20(2) : 210-212.

\section{Introduction:}

A 42-year-old man was admitted to the hospital in internal medicine department with the complaints of low grade fever and gradual weight loss for one year. He also complaints of pigmentation of his skin for last eight months. He states that over the last one year he has had fever which is low grade, intermittent in nature appearing at evening, not associated with chills, rigors and night sweats. During the last one year he has had significant weight loss. For the last eight months he has noticed generalized pigmentation of his skin which is black in color, first involved his face and gradually spread over his whole body. He has not experienced any muscle weakness and pain; he also denies any loose motions, heat intolerance or increased appetite. The patient is a smoker for last 20 years but he is non alcoholic and has no history of any drug abuse. He has been suffering from diabetes type 2 for last 10 years for which has to take oral hypoglycemic drugs. He does not have any medication allergies. His family history is not significant. He suffered from pleural effusion $\&$ pneumothorax one year back and took antituberculosis medications.

On physical examination, the patient is noted to be a thin, slightly emaciated man. His vital signs include a temperature of $101^{\circ} \mathrm{F}$, a pulse of $92 \mathrm{bpm}$, a respiratory rate of 20 breaths / $\mathrm{min}$, a blood pressure of $80 / 60 \mathrm{~mm} \mathrm{Hg}$ (on both standing and lying positions) The patient's sclerae are anicteric. Pigmentation is found all over the body, more marked in face, palmer creases and back of the trunk. There is a scar mark over left knee joint which is not pigmented. The oropharynx is clear but pigmentation is present in the oral mucosa. There are no organomegaly, muscle wasting, proptosis, goiter or palpable lymphnodes. The heart examination reveals a regular rhythm, with no murmurs. The lungs are clear to auscultation in all fields, and no rales or rhonchi are found.

1. Dr. Moniruzzaman Ahmed, Asstt. Professor, Department of Medicine Sylhet MAG Osmani Medical College Hospital

2. Dr. M Ismail Patwary, Professor \& Head, Department of Medicine Sylhet MAG Osmani Medical College Hospital

3. Dr. M A Jalil Ansari, Assc. Professor, Department of medicine, Dhaka Medical College Hospital

4. Dr. Mohammad Saidur Rahman Choudhury, Fellowship Trainee, Department of Medicine Sylhet MAG Osmani Medical College Hospital

Correspondence : Dr. Moniruzzaman Ahmed, Asstt. Professor, Department of Medicine Sylhet MAG Osmani Medical College Hospital 
An abdominal ultrasonogram is taken that shows bilateral adrenal mass, a computed tomography (CT) scan of abdomen was performed which shows bilateral adrenal mass.

His complete blood cell (CBC) count shows a raised ESR $80 \mathrm{~mm} / 1^{\text {st }}$ hour; a normal hemoglobin of $12.10 \mathrm{~g} / \mathrm{dl}$, with a normal white cell count, a normal differential and a normal platelet count. The basic metabolic panel is unremarkable. The free thyroid hormones were within normal range. A tuberculin test was unremarkable. The chest $\mathrm{X}$ - ray and plain $X$ - ray of abdomen did not show any abnormality. Serum electrolytes done repeatedly showed mild hyponatraemia with sodium $<135 \mathrm{mmol} / \mathrm{L} \&$ persistent hyperkalaemia $\left(\mathrm{k}^{+}: 5-5.5 \mathrm{meq} / \mathrm{L}\right)$ and $\mathrm{S}$. cortisol was on lower side of the normal range on several measurements.ACTH stimulation test could not be done due to unavailability of synecthen before treatment. FNAC of adrenal gland was perfoemed in a reputed hospital in Dhaka. The histopathological report of FNAC was suggestive of bilateral adrenal tuberculosis.

The patient was started on four-drug antituberculosis therapy (isoniazid, rifampicin, pyrazinamide and ethambutol) along with hydrocortisone supplementation. Over the next few weeks he showed a marked improvement in constitutional symptoms. On completion of anti tubercular regime repeat CTscan \& USG were done on while the patient was on exogenous glucocorticoid replacement therapy. By this time patient became asymptomatic with marked improvement in skin pigmentation but he developed spontaneous pneumothorax which subsides with conservative treatment.

\section{Discussion}

In 1855, Thomas Addison described autopsy findings of six patients with adrenal tuberculosis, which continues to be one of the most common causes of adrenal insufficiency in the developing world ${ }^{1}$. Guo et al, compared the contrast enhanced CT scan features of the adrenal glands in 42 patients with adrenal tuberculosis. Of these patients, 91\% (38) had bilaterally enlarged adrenals and 51\% (21) had enlarged glands with preservation of the contours $^{2}$. Of these patients, 91\% (38) had bilaterally enlarged adrenals and 51\% (21) had enlarged glands with preservation of the contours $^{2}$.

Clinically inapparent adrenal masses are incidentally detected after imaging studies conducted for reasons other than the evaluation of the adrenal glands. They have frequently been referred to as adrenal incidentalomas ${ }^{3}$.

The presence of an inapparent adrenal mass does not mean absence of endocrine activity. The patient with an adrenal mass requires a complete history and physical examination, biochemical evaluation of all pertinent hormones, and possibly additional radiological studies. Special attention should be given to a history or episodes of high blood pressure, tachycardia, profuse sweating, and to findings such as hirsutism, striae, central obesity, or gynecomastia Diagnostic testing should exclude clinically silent pheochromocytoma, hypercortisolism, and primary aldosteronism. If the diagnosis of overt endocrine disease such as Cushing's syndrome, Conn's syndrome, pheochromocytoma, and congenital adrenal hyperplasia are each suspected during an adrenal mass evaluation, the established diagnostic algorithm for the confirmation and differential diagnosis of these hypersecretory states applies.

The prevalence of chronic primary adrenal insufficiency (Addison's disease) has been reported to be 39 to 60 per million population. The mean age at diagnosis in adult patients is 40 years (range, 17 to 72 ). The most common cause was formerly tuberculous adrenalitis, but now it is autoimmune adrenalitis (slow destruction of the adrenal cortex by cytotoxic lymphocytes), sometimes accompanied by autoimmune thyroid disease and other autoimmune endocrine deficiencies (autoimmune polyglandular syndromes) ${ }^{4}$.

Adrenomyeloneuropathy is an increasingly recognized cause of adrenal insufficiency in young men. It is an X-linked recessive disorder of the metabolism of long-chain fatty acids characterized by spastic paralysis as well as 
adrenal insufficiency; the latter may occur either before or after the neurologic disease ${ }^{5,6}$.

An adrenal insufficiency due to adrenal cortical destruction is the most common endocrine deficiency caused by a Mycobacterium tuberculous infection. Radiographic evidence of adrenal calcification is the most helpful positive diagnostic sign of adrenal tuberculosis, but it is present in only $15 \%$ of cases ${ }^{7}$.

Our patient presented to us with the complaints of prolonged fever for about one year, he had generalized pigmentation, marked weight loss; on abdominal ultrasound he had bilateral adrenal masses. An adrenal insufficiency due to arenocotical destruction is the most common endocrine deficiency caused by a M. tuberculosis infection ${ }^{8}$.

\section{Conclusion:}

Adrenal tuberculosis is a well known cause of primary adrenal insufficiency in developing countries such as Bangladesh. Adrenal mass in tubercular lesion is hardly detected, because by the time patient seeks medical advice the adrenals destruction is complete. This case report presents a case of a patient who presented with fever of unknown origin and bilateral adrenal masses were found as an incidental finding. Early detection in adrenal TB is uncommon. In addition, prolonged fever (PUO) in adrenal mass/tuberculosis is also not widely reported.

\section{References}

1. Mrinal M. Patnaik, MD and Alaka K. Deshpande, MD 2008 Diagnosis-Addison's Disease Secondary to Tuberculosis of the Adrenal Glands. Clinical Medicine \& Research Volume 6, Number 1:29

2. Guo YK, Yang ZG, Li Y, Ma ES, Deng YP, Min PQ, Yin LL, Hu J, Zhang XC, Chen TW. Addison's disease due to adrenal tuberculosis: Contrastenhanced CT features and clinical duration correlation. Eur J Radiol 2006;62:126-131.

3. Georg Mansmann, Joseph Lau, Ethan Balk, Michael Rothberg, Yukitaka Miyachi, Stefan R. Bornstein 2004 The Clinically Inapparent Adrenal Mass: Update in Diagnosis and Management. Endocrine Reviews 25(2):309-340.

4. Wolfgang Oelkers, M.D. Adrenal Insufficiency. The New England Journal of Medicine,1996; 335(16):1206-12

5. Aubourg P. Adrenoleukodystrophy and other peroxisomal diseases. Curr Opin Genet Dev 1994;4:407-11.

6. Blevins LS Jr, Shankroff J, Moser HW, Ladenson PW. Elevated plasma adrenocorticotropin concentration as evidence of limited adrenocortical reserve in patients with adrenomyeloneuropathy. J Clin Endocrinol Metab 1994;78:261-5.

7. Sanford JP, Favour CB (1956) The Interrelationships between Addison's disease and active tuberculosis: A review of 125 cases of Addison's disease. Ann Intern Med 45:56-69.

8. Hyuk-Sang K won, Sang-Il Kim, Soon-Jib Yoo, KunHoo Yoon, Kwang-Woo Lee, Moon-Won Kang, HoYoung Son, Sung-Koo Kang and Bong-Yun Cha 2006 Adrenal Tuberculosis in Cushing's Disease with Bilateral Macronodular Adrenocortical Hyperplasia. Endocrine Journal 53 (2):219-223 